\title{
Ten year survival after heart transplantation: palliative procedure or successful long term treatment?
}

S Fraund, K Pethig, U Franke, T Wahlers, W Harringer, J Cremer, H-G Fieguth, P Oppelt, A Haverich

\begin{abstract}
Objective-To investigate the long term outcome and prognostic factors after heart transplantation.

Setting-University hospital.

Subjects-120 heart transplant patients (98 male, 22 female; underlying disease: dilated cardiomyopathy in 69 , coronary artery disease in 42 , miscellaneous in nine) who had undergone heart transplantation between October 1984 and October 1987. Immunosuppressive treatment was comparable in all patients and rejection episodes were treated in a uniform manner.

Methods-Functional status, quality of life, and potential predictors for long term survival were investigated.

Results-Actuarial survival rates were $65 \%$ at five years and $48 \%$ at 10 years; 58 patients survived $>\mathbf{1 0}$ years. The major causes of death were cardiac allograft vasculopathy $(39 \%)$, acute rejection $(18 \%)$, infection (11\%), and malignancy $(11 \%)$. Long term survivors had good exercise tolerance assessed by the New York Heart Association classification: 47 (81\%) in grade I/II; $11(19 \%)$ in grade III/IV. Echocardiography showed good left ventricular function in 48 patients. On angiography, severe allograft vasculopathy was present in only 16 patients $(28 \%)$. Renal function was only slightly impaired, with mean (SD) serum creatinine of 148.5 (84.9) $\mu \mathrm{mol} / 1$. Multiple potential predictors of long term survival were analysed but none was found useful.

Conclusions-Heart transplantation represents a valuable form of treatment. Survival for more than 10 years with a good exercise tolerance and acceptable side effects from immunosuppression can be achieved in about $50 \%$ of patients.

(Heart 1999;82:47-51)
\end{abstract}

Keywords: heart transplantation; long term survival

Despite recent progress in the conservative treatment of symptomatic heart failure, the clinical course of this disease remains dismal. ${ }^{1-3}$ The introduction of vasodilators and angiotensin converting enzyme (ACE) inhibitors has improved the outcome significantly ${ }^{4-7}$ but mortality remains high. A six month mortality of between $20 \%$ and $30 \%$, as shown in the CONSENSUS (cooperative north Scandina- vian enalapril survival study), cannot be regarded acceptable. ${ }^{6}$

Heart transplantation provides the most effective treatment for patients with end stage heart failure. ${ }^{8}$ Approximately 500 transplantations are performed in Germany each year. With the introduction of cyclosporin as an immunosuppressive agent in the 1980s, marked improvements in survival were achieved in heart transplant programmes. ${ }^{9}$ However, only limited data are available on the long term success of this procedure. We therefore analysed data from patients surviving more than 10 years after heart transplantation and compared them with the results in patients who died before 10 years.

The aim of the study was to assess the long term survival after heart transplantation in a single centre; to characterise quality of life, as defined by exercise tolerance and end organ function; and to identify potential predictors of long term survival.

\section{Methods}

STUDY POPULATION

We studied a cohort of 120 patients (98 male, 22 female) who had undergone heart transplantation at our institution between October 1984 and October 1987. They were followed up closely during the entire period under the Hannover medical school transplant programme until 1997. Baseline demographic data on the patients are summarised in table 1 . The cause of end stage heart failure and the indication for heart transplantation was dilated cardiomyopathy in 69 patients $(57.5 \%)$, coronary artery disease in 42 (35\%), and miscellaneous (valve related disease, hypertrophic obstructive cardiomyopathy, endocardial fibrosis) in nine $(7.5 \%)$. The mean (SD) age of recipients at the time of transplantation was 42.3 (11.8) years, mean donor age was 25.6 (8.7) years, with 83 male and 27 female donors (10 with missing data). Eight patients required retransplantation, five within the first 20 days owing to acute graft failure and another three between 3.0 and 9.1 years after transplantation owing to chronic allograft vascular disease (CAVD).

IMMUNOSUPPRESSION, DRUG TREATMENT, AND MONITORING OF REJECTION

All recipients were treated with a similar immunosuppressive regimen, based on triple therapy using cyclosporin $\mathrm{A}$, prednisolone, and azathioprine. The cyclosporin A target levels
Accepted for publication 18 February 1999 
Table 1 Patient characteristics and evaluation of potential predictors for long term survival

\begin{tabular}{|c|c|c|c|c|}
\hline & $\begin{array}{l}\text { All patients } \\
(n=120)\end{array}$ & $\begin{array}{l}\text { Long term } \\
\text { survivors } \\
(n=58)\end{array}$ & $\begin{array}{l}\text { Deceased } \\
\text { patients } \\
(n=62)\end{array}$ & $p$ Value \\
\hline \multicolumn{5}{|l|}{ Recipient related factors } \\
\hline Age at transplant (years) & $42.3(11.8)$ & $42.1(11.5)$ & $42.5(11.6)$ & 0.8 \\
\hline \multicolumn{5}{|l|}{ Sex } \\
\hline Male & $98(81.7 \%)$ & $49(84.5 \%)$ & $49(79 \%)$ & \multirow[t]{2}{*}{0.4} \\
\hline Female & $22(18.3 \%)$ & $9(15.5 \%)$ & $13(21 \%)$ & \\
\hline \multicolumn{5}{|l|}{ Cause of heart failure } \\
\hline $\mathrm{DCM}$ & $69(57.5 \%)$ & $36(62.1 \%)$ & $33(53.2 \%)$ & \multirow[t]{3}{*}{0.2} \\
\hline CAD & $42(35 \%)$ & $16(27.6 \%)$ & $26(41.9 \%)$ & \\
\hline Others & $9(7 \%)$ & $6(10.3 \%)$ & $3(4.8 \%)$ & \\
\hline Cholesterol at year $1(\mathrm{mmol} / \mathrm{l})$ & $6.7(2.0)$ & $7.0(2.3)$ & $6.4(1.6)$ & 0.1 \\
\hline Triglycerides at year $1(\mathrm{mmol} / \mathrm{l})$ & $2.6(1.8)$ & $2.6(1.9)$ & $2.5(1.4)$ & 0.8 \\
\hline \multicolumn{5}{|l|}{ Donor related factors } \\
\hline Donor age & $25.6(8.7)$ & $23.9(8.8)$ & $26.9(8.4)$ & 0.08 \\
\hline \multicolumn{5}{|l|}{ Donor sex } \\
\hline Male & $83(69.2 \%)$ & $38(65.5 \%)$ & $45(72.6 \%)$ & \multirow[t]{2}{*}{0.9} \\
\hline Female & $27(22.5 \%)$ & $12(20.7 \%)$ & $15(24.2 \%)$ & \\
\hline \multicolumn{5}{|l|}{ CMV mismatch (mismatch/non- } \\
\hline Ischaemic time (min) & $137.6(43.1)$ & $137.2(42.8)$ & $138(43)$ & 0.9 \\
\hline HLA mismatch (47 missing) & $4.6(0.9)$ & $4.6(1.0)$ & $4.6(0.9)$ & 0.8 \\
\hline \multicolumn{5}{|l|}{ Drug treatment } \\
\hline ATG treatment & 99 & 46 & 53 & 0.7 \\
\hline Mean dose of cyclosporin (mg/day) & $327.8(83.0)$ & $318.7(81.8)$ & $338.1(84.0)$ & 0.2 \\
\hline Mean dose of azathioprine (mg/day) & $99.8(30.4)$ & $102(25.5)$ & $97(35.2)$ & 0.4 \\
\hline $\begin{array}{l}\text { Mean dose of prednisolone at year } 1 \\
(\mathrm{mg} / \text { day })\end{array}$ & $10.9(3.4)$ & $10.7(3.7)$ & $10.9(3.0)$ & 0.8 \\
\hline $\begin{array}{l}\text { Number of antihypertensive drugs at } \\
\text { year } 1\end{array}$ & & $1.2(0.9)$ & $1.0(0.9)$ & 0.7 \\
\hline
\end{tabular}

Data are expressed as mean value (SD) and $\mathrm{n}(\%)$. Groups were compared by unpaired two tailed $t$ test for independent values. Non-continuous variables were assessed by $\chi^{2}$ analysis. $\mathrm{p}$ Values $<0.05$ were considered statistically significant.

ATG, antithymocyte globulin; CAD, coronary artery disease; DCM, dilated cardiomyopathy.

were 250 to $300 \mu \mathrm{g} / \mathrm{l}$ for the first year, $150-200$ $\mu \mathrm{g} / 1$ for the second, and then $100-150 \mu \mathrm{g} / 1$ (monoclonal assay). Dose adjustments were determined according to serum creatinine concentrations, maximum values of 120 to 150 $\mu \mathrm{mol} / 1$ being considered acceptable. In 99 patients, inductive cytolytic treatment with antithymocyte globulin (ATG) was given. Al patients were monitored with routine endomyocardial biopsies and echocardiography. Episodes of rejection were treated with a three day course of methylprednisolone (500-1000 $\mathrm{mg}$ /day). Standard drugs were used for the treatment of hypertension, with ACE inhibitors as first choice and calcium channel blockers of the nifedipine type and clonidine used in addition as required. Almost every patient received diuretic treatment with low dose frusemide (furosemide) (20 mg/day).

FOLLOW UP DATA

Patients were seen regularly at the outpatient department at least every three months beyond the first year after transplantation. Quality of life of the surviving patients was assessed, using a standardised questionnaire. Coronary angiographies were performed at one to two year intervals. Cardiac catherisation results were available in the 58 long term survivors and the 31 deceased patients who lived for more than one year after their transplants. Classification of CAVD was as follows: grade 0 , normal angiogram; 1, luminal obstruction $<30 \%$; , $31-50 \% ; 3,>50 \% ; 4$, complete closure of the vessel.

ANALYSIS OF PREDICTIVE FACTORS

Analysis of predictive factors was performed comparing characteristics of long term survi-

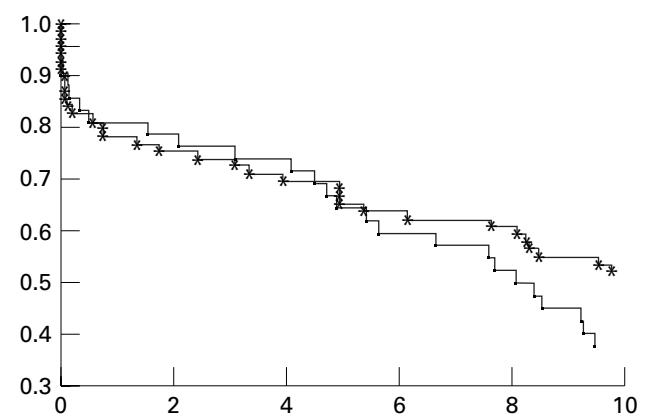

Figure 110 year actuarial survival rates computed by Kaplan-Meier estimate: * — *, dilated cardiomyopathy as the underlying disease; $\bullet-\bullet$ coronary artery disease as the underlying disease.

vors with deceased patients. The following variables were analysed: recipient age, donor age, sex, cytomegalovirus (CMV) status, HLA mismatch (available in 73 cases), lipid levels at year 1 , ischaemic time, ATG induction treatment, mean dose/day of cyclosporin A, azathioprine, and prednisolone at year 1 , and the number of antihypertensive drugs at year 1 .

DATA ANALYSIS

Data analysis was performed using a computer assisted software package (SPSS, version 6.0.1). Continuous variables are presented as mean (SD). Univariate comparison between surviving and deceased patients for different risk factors was performed by $\chi^{2}$ test for non-continuous variables and by unpaired two tailed $t$ test for continuous values. For multivariate analysis, a logistic regression was chosen (backward, LR). Probability (p) values $<0.05$ were considered significant. Actuarial survival rates were computed according to the Kaplan-Meier estimate using the log rank test.

\section{Results}

\section{SURVIVAL}

Of the total cohort, 58 patients survived more than 10 years, with an actuarial survival rate of $78.3 \%$ at 1 year, $65 \%$ at 5 years, and $48 \%$ at 10 years (fig 1).

There were 62 deaths among the study population during the follow up period. Causes of death in the entire study group are given in fig $2 \mathrm{~A}$. The most common causes of death were chronic graft failure owing to CAVD ( $n=24$; $39 \%)$, early right heart failure ( $n=11 ; 18 \%)$, infections $(\mathrm{n}=7 ; 11.2 \%)$, and malignancies $(n=7 ; 11.2 \%)$. Forty per cent of the deaths occurred within the first year of transplantation, mainly from acute graft rejection. Causes of death in year 1 are shown in fig $2 \mathrm{~B}$.

GRAFT FUNCTION AND SIDE EFFECTS OF IMMUNOSUPPRESSION

In the study population, 58 patients $(48.3 \%)$ survived the 10 year period, with a mean observation period of 11.1 (0.7) years. Assessment of the quality of life by the self assessment questionnaire was optimistic in about $75 \%$ of the patients. Only two patients were seriously dissatisfied. To the question "Would you decide to have a heart transplant again?" only five gave a negative response. Exercise toler- 
A

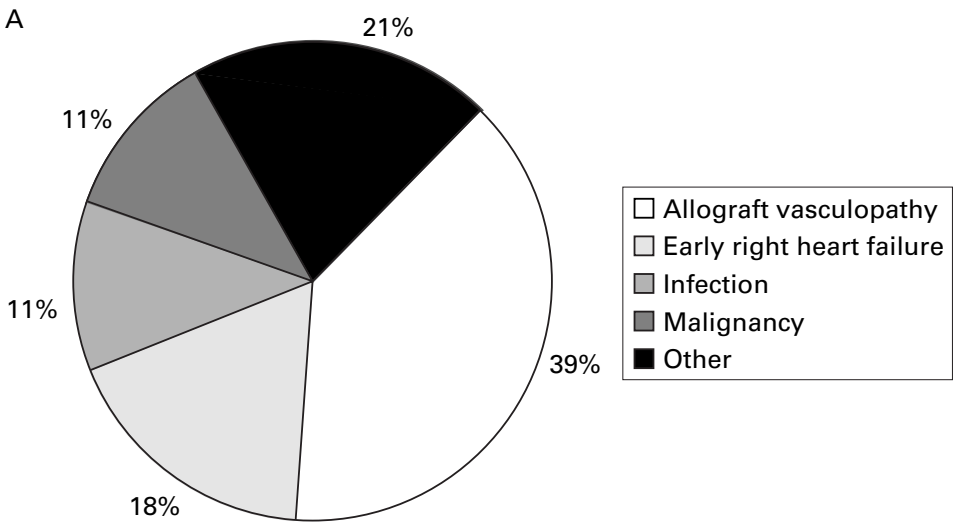

B

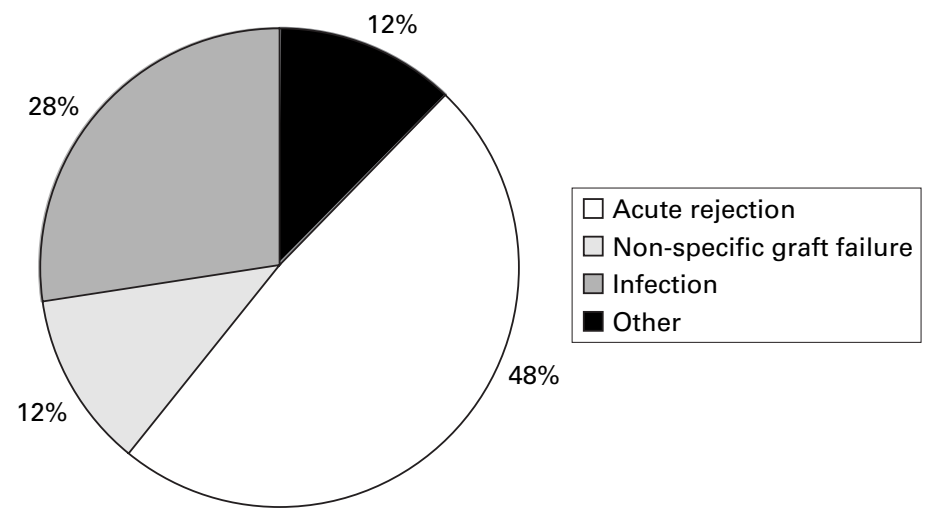

Figure 2 (A) Causes of death in group as a whole; (B) causes of death in year 1.

ance in long term survivors could be shown to be excellent, with 14 patients $(24.1 \%)$ being in New York Heart Association (NYHA) functional class I, $33(56.9 \%)$ in class II, nine $(15.5 \%)$ in class III, and two $(3.4 \%)$ in class IV, as assessed by routine visits to the outpatient department. Normal graft function was demonstrated by echocardiographic quantification of left ventricular contractility, with a mean (SD) ejection fraction of 61 (5)\%.

As an index of end organ impairment we assessed renal function, defined by serum creatinine. The mean serum creatinine was 148.5 (84.9) $\mu \mathrm{mol} / 1$, showing an acceptable functional status, with only one patient requiring chronic haemodialysis. Table 2 gives the data for this group.

\section{ALLOGRAFT VASCULOPATHY}

The most recent coronary angiography in the survivors showed no signs of graft vasculopathy in 27 patients $(46.6 \%), 15(25.8 \%)$ had a luminal obstruction of grade 1 or 2 , and only $16(27.6 \%)$ had high grade vasculopathy. Coronary angiograms were available in 31 deceased patients; in 13 cases $(41.9 \%)$ a luminal obstruction of $50 \%$ and more was detectable, as compared with $16 / 58(27.6 \%)$ in long term survivors. In addition, the latency until the first sign of graft vasculopathy $(<30 \%$ luminal obstruction) diagnosed in this group was 40.9 (31.6) months, whereas the latency in the long term surviving group was 76.4 (28.5) months.
Table 2 NYHA classification and organ function of long term survivors

\begin{tabular}{|c|c|}
\hline & $n(\%)$ \\
\hline \multicolumn{2}{|l|}{ Exercise tolerance } \\
\hline NYHA class I & $14(24.1)$ \\
\hline class II & $33(56.9)$ \\
\hline class III & $9(15.5)$ \\
\hline class IV & $2(3.5)$ \\
\hline \multicolumn{2}{|l|}{ Allograft vasculopathy } \\
\hline CAVD 0 & $27(46.6)$ \\
\hline 1 & $9(15.5)$ \\
\hline 2 & $6(10.3)$ \\
\hline 3 & $15(25.9)$ \\
\hline 4 & $1(1.7)$ \\
\hline \multicolumn{2}{|l|}{ Graft/organ function } \\
\hline Ejection fraction (\%) & $61(5)$ \\
\hline Mean creatinine $(\mu \mathrm{mol} / \mathrm{l})$ & $148.5(84.9)$ \\
\hline Non-cutaneous malignancy & $8(13.7)$ \\
\hline
\end{tabular}

Classification of CAVD: grade 0 , normal angiogram; 1 , luminal obstruction $<30 \% ; 2,31-50 \% ; 3,>50 \% ; 4$, complete closure of the vessel.

NYHA, New York Heart Association.

MALIGNANCY

There was a high incidence of malignant tumours in both the deceased patients and the long term survivors. In the overall cohort, 17 patients $(14.1 \%)$ developed non-cutaneous malignant tumours, with lymphoma being the commonest (nine cases, $52.9 \%$ of all tumours). Malignancy was the cause of death in $11 \%$ of the deceased patients. However, malignant disease was found in the long term survivors as well (eight cases $(13.7 \%)$ : lymphoma in three, lung cancer in two, colon cancer in one, and haematological neoplasia in one), five of these being in complete remission after successful treatment at the time of writing.

\section{ANALYSIS OF PREDICTIVE FACTORS}

Analysis of predictive factors was performed comparing characteristics of the long term survivors and the deceased patients. Using both a univariate analysis and a multivariate logistic regression, no independent predictors of long term survival for more than 10 years could be identified. The data on factors relating to recipient, donor, procedure, and treatment are summarised in table 1 . There was no significant difference between the two groups. There was a trend for a higher proportion of patients with dilated cardiomyopathy to survive more than 10 years after heart transplantation; this resulted in a 10 year survival rate of $38 \%$ for coronary artery disease versus $55 \%$ for dilated cardiomyopathy (NS, log rank test).

\section{Discussion}

During the past 25 years considerable progress has been made in understanding the pathogenesis of the heart failure syndrome. On the basis of this knowledge several advances have been made in the conservative management in this patient group. ${ }^{5610}$ However, despite the introduction of vasodilators and ACE inhibitors, one year mortality still ranges from $15 \%$ among unselected patients to $50 \%$ among those in NYHA functional class IV. ${ }^{67}$

In contrast, heart transplantation allows one year and five year survival rates of about $80 \%$ and $60 \%$ to $70 \%$, respectively. ${ }^{11}$ However, with more than 30 years of clinical experience with replacement of the heart, only limited data are 
available in allograft recipients surviving 10 years and more. We therefore analysed a patient population undergoing heart transplantation more than 10 years earlier to characterise survival, graft function, and side effects of immunosuppression in the hope of identifying potential predictors of long term survival.

Our study represents a single centre approach with a 10 year survival rate of $48 \%$, compared with $45 \%$ reported by the multicentre International Society for Heart and Lung Transplantation registry. These results are far superior to medical treatment alone. Before the introduction of vasodilators and ACE inhibitors, a one year mortality of approximately $50 \%$ was the norm with conservative management. ${ }^{12}$ In the CONSENSUS trial, in which patients with severe heart failure were randomised to receive placebo or enalapril in addition to conventional treatment, the mortality at 12 months was $36 \%$ in the enalapril group and $52 \%$ in the placebo group. ${ }^{6}$ In the V-Heft I (vasodilator in heart failure) study, which included patients with mild to moderate clinical heart failure, the cumulative mortality over four years was $53.6 \%$. $^{7}$

However, quality of life is also a major issue after heart transplantation. In the CONSENSUS study, $42 \%$ of the enalapril treated patients had improvement in their NYHA functional class, but three quarters of the surviving patients remained in NYHA functional class III and IV. In our transplant population the majority of patients $(81 \%)$ were in NYHA functional class I and II as long as 10 years after the procedure. Exercise tolerance proved to be remarkably improved compared with optimum medical treatment. Furthermore, invasive and non-invasive evaluation of graft function by cardiac catherisation and echocardiography revealed normal cardiac function with a mean ejection fraction of 61 (5)\%. These results are in agreement with the findings of previous investigations. ${ }^{13}{ }^{14}$ In 1993, von Scheidt evaluated the long term haemodynamic profile of heart transplant recipients up to seven years after the procedure and reported essentially normal systolic function with no evidence of the development of a dilated or restrictive post-transplantation cardiomyopathy over time. ${ }^{14}$

Apart from good functional graft status, accelerated graft vasculopathy was identified as one of the most critical issues in the long term follow up of heart transplant recipients. ${ }^{15-18}$ This unique form of an accelerated coronary syndrome is characterised by endothelial dysfunction and a multifocal intimal hyperplasia modified by coronary artery remodelling. ${ }^{19-21}$ The prevalence of this complication-as assessed by angiography-is approximately $10 \%$ to $15 \%$ at one year and $35 \%$ to $50 \%$ five years after transplantation. ${ }^{22}{ }^{23}$ Recent reports were also able to show positive effects on the development of CAVD by lipid lowering treatment with statins as well as by diltiazem administration. ${ }^{24}{ }^{25}$ Because of the retrospective design of our study, comprising patients transplanted between 1984 and 1987, only a small minority received these agents. Future pro- spective studies will have to evaluate whether the introduction of these drugs (in 1994/95) has resulted in clinical benefit.

The clinical impact of CAVD on heart transplantation was confirmed in our patient cohort. CAVD was the major cause of death in $39 \%$ of the patients. In deceased patients the first signs of CAVD ( $30 \%$ luminal obstruction) were diagnosed significantly earlier than in long term survivors (after 40.9 (31.6) months $v 76.4$ (28.5) months, $\mathrm{p}<0.05)$. Peak incidence of death from CAVD was between five and 10 years after heart transplantation. Most interestingly, the majority of long term survivors presented with only low grade vasculopathy ( $72.4 \%$ less than grade 2$)$, suggesting a positive selection in this subgroup and raising hopes for a stable subsequent course.

Renal function in our cohort showed only moderate impairment, with a mean serum creatinine of 148.5 (84.9) $\mu \mathrm{mol} / 1$ and only one patient requiring chronic haemodialysis. This important finding suggests that chronic damage to the kidneys caused by long term treatment with cyclosporin A can be minimised by optimal adjustment of the dose, using toxicity adjusted drug level monitoring. As shown by Hausen et al, the concept of cyclosporin A dose adjustment governed primarily by renal function does not affect the incidence of rejection. ${ }^{26}$

Finally, the development of malignancy remains a point of major concern as an important cause of death in long term survivors after any type of solid organ transplantation, as well as in heart transplantation..$^{27}$ Nearly $25 \%$ of our patients suffered from malignant disease during the 10 year period $(13.7 \%$ of long term survivors and $11.2 \%$ dying from malignancy). This represents one of the most challenging problems for future attempts to optimise the immunosuppressive protocols. Induction treatment with OKT 3, a major determinant of lymphoma incidence, was not used in our cohort during the study period. ATG was used as cytolotytic induction treatment in 99 patients, with no significant difference between survivors and non-survivors.

Searching for predictive factors for survival, Bourge et al reviewed the data from the cardiac transplant research database established in $1990 .^{29}$ Analysing short term periods, they identified different recipient (younger and older age), donor (older age, smaller body surface area), and clinical (longer ischaemic time) risk factors for death after transplantation. However, evaluation of different recipient, donor, and treatment related factors in our cohort showed no significant influence on long term outcome. The incidence of HLA mismatch, which was shown to correlate with early allograft rejection incidence and graft survival by De Mattos et al and Smith et al, ${ }^{30}{ }^{31}$ was not different in our survivor and non-survivor groups, though complete data were only available in 73 patients. We only found a positive trend in patients with dilated cardiomyopathy as the underlying disease, which might reflect the younger age of the recipients $(39.1$ (12.2) $v$ 
48.2 (5.8) years), less concomitant disease, and better general physical condition in this subgroup.

In summary, the results of cardiac transplantation have improved significantly over the last two decades, with an increasing number of patients surviving between 10 and 20 years after transplantation. As we were able to show in our population, nearly $50 \%$ of patients will survive a 10 year period with acceptable exercise tolerance and quality of life. Thus heart transplantation should be regarded as a valuable treatment option. The major obstacle for the future of these patients will be to overcome chronic graft vasculopathy and the development of malignancies.

1 Franciosa JA, Wilen M, Ziesche S, et al. Survival in men with severe chronic left ventricular failure due to either coronary
heart disease or idiopathic dilated cardiomyopathy. $A m \mathcal{F}$ heart disease or idiopathic
Cardiol 1983;51:831-6.

2 Fuster V, Gersh B, Giuliani E, et al. The natural history of diopathic dilated cardiomyopathy. Am f Cardiol 1983;51: 831-6.

3 McKee PA, Castelli WP, McNamara PM, et al. The natural history of congestive heart failure: the Framingham study. N Engl F Med 1971;285:1441-6.

4 Cohn JN, Franciosa JA. Vasodilator therapy of cardiac failure. N Engl f Med 1977; 297:27-31;254-8.

5 Cohn JN, Archibald DG, Ziescher S, et al. Effect of vasodilator therapy on mortality in chronic congestive heart failure: results of a Veterans Administration cooperative failure: results of a Veterans Administration coop

6 The CONSENSUS Trial Study Group. Effects of enalapril on mortality in severe congestive heart failure. Results of on mortality in severe congestive heart failure. Results of (CONSENSUS). N Engl f Med 1987;316:1429-35.

7 Cohn JN, Johnson G, Ziesche S, et al. A comparison of enalapril with hydralazine-isosorbide dinitrate in the treatenalapril with hydralazine-isosorbide dinitrate in the treatment of chronic

8 O'Connell JB, Bourge RC, Costanzo-Nordin MR, et al. Cardiac transplantation: recipient selection, donor procurement, and medical follow up. A statement for health professionals from the Committee on Cardiac Transplantation of the Council on Clinical Cardiology, American Heart Association. Circulation 1992;86:1061-79.

9 Oyer PE. Heart transplantation in the cyclosporine era. Ann Thorac Surg 1988;46:489-90.

10 Packer M, Carver JR, Rodeheffer RJ, et al. Effect of oral milrinone on mortality in severe chronic heart failure. The PROMISE Study Research Group. $N$ Engl $\mathcal{f}$ Med 1991;325:1468-75

11 Hosenpud JD, Novick RJ, Bennett LE, et al. The registry of the International Society for Heart and Lung Transplantation: official report-1996. $\mathcal{F}$ Heart Lung Transplant 1996;15:655-74.

12 Smith WM. Epidemiology of congestive heart failure. $A m \mathcal{F}$ Cardiol 1985;55(suppl):3-8.
13 Frist WH, Stinson EB, Oyer PE, et al. Long-term hemodynamic results after cardiac transplantation. 7 Thorac namic results after cardiac tr
Cardiovasc Surg 1987;94:685-93.

14 von Scheidt W, Ziegler B, Kemkes BM, et al. Long-term myocardial function after heart transplantation. Thorac Cardiovasc Surg 1993;41:156-62.

15 Gao SZ, Aldermann EL, Schroeder JS, et al. Accelerated coronary vascular disease in the heart transplant patient: coronary arteriography findings. $7 \mathrm{Am}$ Coll Cardiol 1988; 12:334-40

16 Johnson DE, Gao SZ, Schroeder JS, et al. The spectrum of coronary artery pathologic findings in human cardiac allografts. F Heart Transplant 1989;8:349-59.

17 Schroeder JS, Hunt SA. Cardiac transplantation: where are we? N Engl f Med 1986;315:961-3.

18 Miller LW. Allograft coronary artery disease. Heart Failure 1989;5:253-9.

19 Klauss V, Ackermann K, Hennecke KH, et al. Epicardial intimal thickening in transplant coronary artery disease and resistance vessel response to adenosine. Circulation 1997;96(suppl II):II-1564.

20 Chenzbraun A, Pinto F, Alderman E, et al. Distribution and morphologic features of coronary artery disease in cardiac allografts: an intracoronary ultrasound study. $\mathcal{f} \mathrm{Am}$ Soc Echocardiogr 1995;8:1-8.

21 Pethig K, Heublein B, Wahlers T, et al. Mechanism of luminal narrowing in cardiac allograft vasculopathy: inadequate remodeling rather than intimal hyperplasia is the major predictor of coronary artery stenosis. Am Heart $\mathcal{f}$ 1998;135:628-33.

22 Gao SZ, Schroeder J, Alderman E, et al. Incidence of accelerated coronary artery disease in heart transplant survivors: comparison of cyclosporine and azathioprine regimen [abstract]. Circulation 1988;78(suppl II):280.

23 Ratkovec RM, Wray RB, Renlund DG, et al. Influence of corticosteroid-free maintenance immunosuppression in corticosteroid-free maintenance immunosuppression in tion. $\mathcal{F}$ Thorac Cardiovasc Surg 1990;100:6-12.

24 Kobashigawa JA, Katznelson S, Laks H, et al. Effect of pravastatin on outcome after cardiac transplantation. $N$ Engl $\mathcal{F}$ Med 1995; 333:621-7.

25 Schroeder JS, Gao SZ, Alderman EL, et al. Prevention of transplant accelerated coronary vascular disease with diltiazem. F Am Coll Cardiol 1994;91:1647-54.

26 Hausen B, Demertzis S, Rohde R, et al. Low-dose cyclosporine therapy in triple-drug immunosuppression for heart transplant recipients. Ann Thorac Surg 1994;58:9991004

27 Penn I. Incidence and treatment of neoplasia after transplantation. 7 Heart Lung Transplant 1993;12:328-36.

28 Pethig K, Fischer H, Wahlers T, et al. Todesursachen nach Herztransplantation: Einfluss auf die klinische Langzeitbetreuung. Z Kardiol 1997;86:S3,98.

29 Bourge CR, Kirklin JK, Naftel DC, et al. Predicting outcome after cardiac transplantation: lessons from the cardiac transplant research database. Curr Opin Cardiol 1997;12:136-45.

30 De Mattos AM, Head MA, Everett J, et al. HLA-DR mismatching correlates with early cardiac allograft rejection, incidence, and graft survival when high confidence level serological DR typing is used. Transplantation 1994;54:626-30.

31 Smith DJ, Rose ML, Pomerance A, et al. Reduction of cellular rejection and increase in longer-term survival after heart transplantation after HLA-DR matching. Lancet 1995;346:1318-22. 